УДК 517.926, 517.977.1

\title{
Local R-observability of Differential-algebraic Equations
}

Pavel S. Petrenko*

Matrosov Institute for System Dynamics and Control Theory of SB RAS

Lermontov, 134, Irkutsk, 664033

Russia

Received 21.12.2015, received in revised form 05.02.2016, accepted 01.05.2016

A nonlinear system of first order ordinary differential equations is considered. The system is unresolved with respect to the derivative of the unknown function and it is identically degenerate in the domain. An arbitrarily high unresolvability index is admited. Analysis is carried out under assumptions that ensure the existence of a global structural form that separates "algebraic" and "differential" subsystems. Local $R$-observability conditions are obtained by linear approximation of the system.

Keywords: local observability, differential-algebraic equation, observable nonlinear system. DOI: 10.17516/1997-1397-2016-9-3-353-363.

\section{Introduction and preliminaries}

Let us consider the system of nonlinear ordinary differential equations (ODEs)

$$
\begin{gathered}
F\left(t, x(t), x^{\prime}(t)\right)=0, \quad t \in I=\left(a_{0}-\varepsilon, a_{0}+\varepsilon\right), \\
y(t)=\phi(t, x),
\end{gathered}
$$

where $\operatorname{det} \frac{\partial F\left(t, x, x^{\prime}\right)}{\partial x^{\prime}} \equiv 0, F\left(t, x, x^{\prime}\right)$ is a sufficiently smooth function with values in $\mathbf{R}^{n}$ and domain $\mathcal{D}=I \times \mathcal{X} \times \mathbf{R}^{n}, \mathcal{X}$ is a neighborhood of the point $x=0 ; \phi: I \times \mathcal{X} \rightarrow \mathbf{R}^{m}(m<n)$ is a continuous function such that $\phi(t, 0)=0 \quad \forall t \in I ; x(t)$ is the $n$-dimensional function of state; $a_{0} \in \mathbf{R} ; \varepsilon=$ const $>0 ; \phi^{\prime}(t)=\frac{d}{d t} \phi(t), \phi^{(i)}(t)=\left(\frac{d}{d t}\right)^{i} \phi(t) \forall \phi(t) \in \mathbf{C}^{i}(I)$.

Such systems are called differential-algebraic equations (DAEs). The measure of unresolvability for the DAEs with respect to the derivative is an integer value $r: 0 \leqslant r \leqslant n$, which is called index [1, pp. 16-17].

Analysis is carried out under assumptions that function $F$ has the property

$$
F(t, 0,0)=0 \quad \forall t \in I .
$$

Algebraic conditions of the full observability and $R$-observability based on the reduction to the Kronecker canonical form were obtained for the linear DAEs with constant coefficients and regular matrix pencil [2, pp. 29-44]. The local observability of the ODEs systems resolved with respect to the derivative was investigated in [3, pp. 355-370]. The local observability based on normalization of the DAEs by so-called extended system is investigated in [4].

In this paper, to the best of our knowledge, the local $R$-observability of the nonlinear DAEs (1.1) is first investigated with the use of the first approximation system. The local $R$-observability of system (1.1) means that there is the only one way to restore a consistent initial state of the system via the specified output function.

The analysis is carried out under assumptions that ensure the existence of a global structural form that separates "algebraic" and "differential" subsystems. They have the same solutions as the original system $[5,6]$.

* petrenko_p@mail.ru

(c) Siberian Federal University. All rights reserved 


\section{Definitions}

Definition 1 ( [6], p. 58). The system of finite equations

$$
\mathcal{F}_{r}\left(t, x, y, z_{1}, \ldots, z_{r}\right)=\left(\begin{array}{c}
F(t, x, y) \\
F_{1}\left(t, x, y, z_{1}\right) \\
\ldots \\
F_{r}\left(t, x, y, z_{1}, \ldots, z_{r},\right)
\end{array}\right)=0
$$

where $x, y, z_{j} \in \mathbf{R}^{n}$, and functions $F_{j}\left(t, x, y, z_{1}, \ldots, z_{j}\right)(j=\overline{1, r})$ have the property: for any $n$-dimensional vector-function $\phi(t) \in \mathbf{C}^{j+1}(I)$ such that $\left(t, \phi(t), \phi^{\prime}(t)\right) \in \mathcal{D} \quad \forall t \in I$,

$$
F_{j}\left(t, \phi(t), \phi^{\prime}(t), \phi^{\prime \prime}(t), \ldots, \phi^{(j+1)}(t)\right)=\left(\frac{d}{d t}\right)^{j} F\left(t, \phi(t), \phi^{\prime}(t)\right),
$$

is called r-derivative array equations of system (1.1).

The following objects are associated with function $F(t, x, y)$ :

$$
\Gamma_{r, z}=\left(\begin{array}{lll}
\partial \mathcal{F}_{r} / \partial z_{1} & \ldots & \partial \mathcal{F}_{r} / \partial z_{r}
\end{array}\right), \quad \Gamma_{r, y}=\left(\begin{array}{lll}
\partial \mathcal{F}_{r} / \partial y & \Gamma_{r, z}
\end{array}\right), \quad \Gamma_{r, x}=\left(\begin{array}{ll}
\partial \mathcal{F}_{r} / \partial x & \Gamma_{r, y}
\end{array}\right) .
$$

These are matrices with dimensions $n(r+1) \times n r, n(r+1) \times n(r+1)$ и $n(r+1) \times n(r+2)$, respectively

In accordance with assumption (1.3) the point $t=a_{0}, x=y=z_{j}=0,(j=\overline{1, r})$ satisfies the r-derivative array equations (2.1). We denote this point as $\alpha_{r}=\left(a_{0}, 0, \ldots, 0\right)$. If $\operatorname{rank} \Gamma_{r, x}\left(\alpha_{r}\right)=$ $n(r+1)$ then system (2.1) satisfies all the assumptions of the implicit function theorem [7, c. 66]. According to this theorem, $n(r+1)$ components of the vector colon $\left(x, y, z_{1}, \ldots, z_{r}\right)^{\dagger}$ can be expressed from (2.1). Let us denote them by $\xi$. Other $n$ components of this vector is denoted by $\eta$. Components $\xi$ depend on $t$ and $\eta$ :

$$
\xi=\xi(t, \eta),(t, \eta) \in \mathcal{W},
$$

where $\mathcal{W}=I_{0} \times \tilde{\mathcal{W}} ; I_{0}=\left(a_{0}-\varepsilon_{0}, a_{0}+\varepsilon_{0}\right) \subseteq I, 0<\varepsilon_{0} \leqslant \varepsilon ; \tilde{\mathcal{W}} \subset \mathbf{R}^{n}$ is a neighborhood of the point $\eta=0$;

$$
\operatorname{colon}(\xi, \eta)=P \operatorname{colon}\left(x, y, z_{1}, \ldots, z_{r}\right),
$$

$\xi \in \mathbf{R}^{n(r+1)}, \eta \in \mathbf{R}^{n}, P$ is the row permutation matrix [8, pp. 127-128].

Since matrix $\Gamma_{r, x}$ has dimensions $n(r+1) \times n(r+2)$ then in general case the non-special minor of $n(r+1)$ order of matrix $\Gamma_{r, x}\left(\alpha_{r}\right)$ is not unique. The implicit function determined in $(2.2)$ depends on this minor. We find the minor in the following way. We choose $\varrho=\operatorname{rank} \Gamma_{r, y}\left(\alpha_{r}\right)(\varrho \leqslant$ $n(r+1))$ linearly independent columns of matrix $\Gamma_{r, y}\left(\alpha_{r}\right)$. We should include the maximum possible number of the first $n$ columns of this matrix. We add this columns to $n(r+1)-\varrho$ linearly independent columns of the matrix $\partial \mathcal{F}_{r} / \partial x$ at the point $\alpha_{r}$ which represent the first $n$ columns of the matrix $\Gamma_{r, x}\left(\alpha_{r}\right)$. These $n(r+1)$ linearly independent columns represent the required minor. As this takes place, $\varrho \geqslant n r$.

Definition 2 ( [6], p. 59). The obtained above non-special minor of $n(r+1)$ order of the matrix $\Gamma_{r, x}\left(\alpha_{r}\right)$ is called resolving minor.

We assume that functions (2.2) correspond to the resolving minor. The matrix which obtained by substitution of functions $(2.2)$ into the matrix $\Gamma_{r, z}\left(t, x, y, z_{1}, \ldots, z_{r}\right)$ is denoted by $\bar{\Gamma}_{r, z}(t, \eta)$.

\footnotetext{
${ }^{\dagger} \operatorname{colon}\left(c_{1}, c_{2}, \ldots, c_{n}\right)=\left(\begin{array}{llll}c_{1}^{\top} & c_{2}^{\top} & \ldots & c_{n}^{\top}\end{array}\right)^{\top}$; symbol $\top$ means transpose.
} 


\section{Equivalent structural form}

\subsection{Nonlinear DAEs}

Let $F(t, x, y) \in \mathbf{C}^{r+1}(\mathcal{D})$. In addition the following conditions are satisfied:

A) $\mathcal{F}_{r}\left(\alpha_{r}\right)=0, \operatorname{rank} \Gamma_{r, x}\left(\alpha_{r}\right)=n(r+1)$;

B) $\operatorname{rank} \bar{\Gamma}_{r, z}(t, \eta)=\rho=$ const everywhere in the domain $\mathcal{W}$;

C) resolving minor of the matrix $\Gamma_{r, x}\left(\alpha_{r}\right)$ includes $\rho$ columns of the matrix $\Gamma_{r, z}\left(\alpha_{r}\right)$ and the first $n$ columns of the matrix $\Gamma_{r, y}\left(\alpha_{r}\right)$.

Definition 3 ( [6], p. 60). The smallest integer $r$, for which conditions A), B), C) are satisfied, is called the index of DAEs (1.1).

Definition 4. $N$-dimensional vector-function $x_{*}(t) \in \mathbf{C}^{1}(I)$ is called the solution of the system (1.1) if it turns this system identically on I.

It was shown [6, p.61] that under certain assumptions DAE (1.1) has the same solutions as the system

$$
\begin{gathered}
x_{1}^{\prime}(t)=f_{1}\left(t, x_{1}(t)\right), \\
x_{2}(t)=f_{0}\left(t, x_{1}(t)\right), \quad t \in I_{0},
\end{gathered}
$$

where functions $f_{1}, f_{0}$ are defined in $\mathcal{W}$. They have continuous derivatives in $\mathcal{W}$ with respect to their arguments and $x_{1} \in \mathbf{R}^{n-d}, x_{2} \in \mathbf{R}^{d}$;

$$
\operatorname{colon}\left(x_{1}(t), x_{2}(t)\right)=Q x(t),
$$

where $Q$ is the row permutation matrix. There is also a method of constructing system (3.1), (3.2) [6, p.61]. The system was obtained from components of implicit function (2.2) that satisfy $r$-derivative array equations (2.1).

Then in view of (3.3), output function (1.2) can be rewritten as

$$
y(t)=\tilde{\phi}\left(t, Q^{-1} \operatorname{colon}\left(x_{1}(t), x_{2}(t)\right)\right)=\tilde{\phi}\left(t, Q^{-1} \operatorname{colon}\left(x_{1}(t), f_{0}\left(t, x_{1}(t)\right)\right)\right)=\left(\begin{array}{c}
\phi_{1}\left(t, x_{1}(t)\right) \\
\phi_{2}\left(t, x_{1}(t)\right)
\end{array}\right),
$$

where the function

$$
y_{1}(t)=\phi_{1}\left(t, x_{1}(t)\right)
$$

is an output function corresponding to system (3.1).

Let us fix the point $t_{0} \in I_{0}$, define the following initial conditions for DAEs (3.1), (3.2)

$$
x_{1}\left(t_{0}\right)=x_{1,0}, \quad x_{2}\left(t_{0}\right)=x_{2,0}
$$

and assume that the equality

$$
x_{2,0}=f_{0}\left(t_{0}, x_{1,0}\right)
$$

is satisfied.

If problem (3.1), (3.2), (3.6) has on some interval $I_{\tau}=\left(t_{0}-\tau, t_{0}+\tau\right) \subseteq I_{0} \quad(\tau>0)$ a solution $x_{*}(t)=Q^{-1} \operatorname{colon}\left(x_{*, 1}(t), x_{*, 2}(t)\right)$ then inclusion $\left(x_{*}(t), x_{*}^{\prime}(t)\right) \in \mathcal{V} \quad \forall t \in I_{\tau}$ should be satisfied, where $\mathcal{V} \subset \mathbf{R}^{2 n}$ is a neighborhood of the point $x=0, y=0$. Then norms of vectors $x_{1,0}, x_{2,0}$ in (3.6) should be sufficiently close to zero.

The following theorem is a sufficient condition for the local solvability of Cauchy problem

$$
x\left(t_{0}\right)=z_{0}
$$

$\left(z_{0} \in \mathbf{R}^{n}\right)$ for DAE (1.1) [6]. 
Theorem 1. Let us assume that

1) $F(t, x, y) \in \mathbf{C}^{r+2}(\mathcal{D})$;

2) $\mathcal{F}_{r+1}\left(\alpha_{r+1}\right)=0$ and assumptions $\left.\left.\left.A\right), B\right), C\right)$ are satisfied;

3) $\operatorname{rank} \Gamma_{r+1, y}\left(\alpha_{r+1}\right)=\operatorname{rank} \Gamma_{r, y}\left(\alpha_{r}\right)+n$.

Then $\forall t_{0} \in I_{0}$ there is $\delta>0$ and $\tau=\tau\left(t_{0}\right)>0$ such that for any vector $z_{0} \in \mathbf{R}^{n}$ with $\left\|z_{0}\right\|<\delta$, a solution $x_{*}(t) \in \mathbf{C}^{2}\left(I_{\tau}\right)$ to problem (3.1), (3.2), (3.8) is defined on the interval $I_{\tau}$, where $z_{0}=Q^{-1}$ colon $\left(x_{1,0}, x_{2,0}\right) \ddagger$. Wherein $x_{*}(t)$ is a solution of problem (1.1), (3.8) on $I_{\tau}$.

In what follows, without loss of generality, we assume that $t_{0}=a_{0}$ and $I_{\tau}=I_{0}$, i.e. $\tau=\varepsilon_{0}$.

Definition 5 ( [6], p.61). DAEs (3.1), (3.2) are called the equivalent form for system (1.1) in the neighborhood $\mathcal{A}$ of the point $\alpha_{r}$.

Definition 6 ( [5], p.78). The initial condition (3.8) which satisfies (3.7) is called consistent with system (1.1), where $\operatorname{colon}\left(x_{1,0}, x_{2,0}\right)=Q z_{0}$.

\subsection{Linear DAEs}

Property (1.3) allows us to determine the matrices $A(t)=\frac{\partial F}{\partial y}(t, 0,0), \quad B(t)=\frac{\partial F}{\partial x}(t, 0,0)$. Then the DAE

$$
A(t) x^{\prime}(t)+B(t) x(t)=0, \quad t \in I,
$$

is the first approximation for (1.1). Function

$$
y(t)=C(t) x(t)
$$

is the linear approximation for $(1.2)$, where $C(t)=\frac{\partial \phi}{\partial x}(t, 0)$.

Matrices

$$
\begin{aligned}
& \mathrm{D}_{r, z}(t)=\left(\begin{array}{cccc}
C_{1}^{1} A(t) & O & \ldots & O \\
C_{2}^{1} A^{\prime}(t)+C_{2}^{2} B(t) & C_{2}^{2} A(t) & \ldots & O \\
\vdots & \vdots & \ddots & \vdots \\
C_{r}^{1} A^{(r-1)}(t)+C_{r}^{2} B^{(r-2)}(t) & C_{r}^{2} A^{(r-2)}(t)+C_{r}^{3} B^{(r-3)}(t) & \ldots & C_{r}^{r} A(t)
\end{array}\right),
\end{aligned}
$$

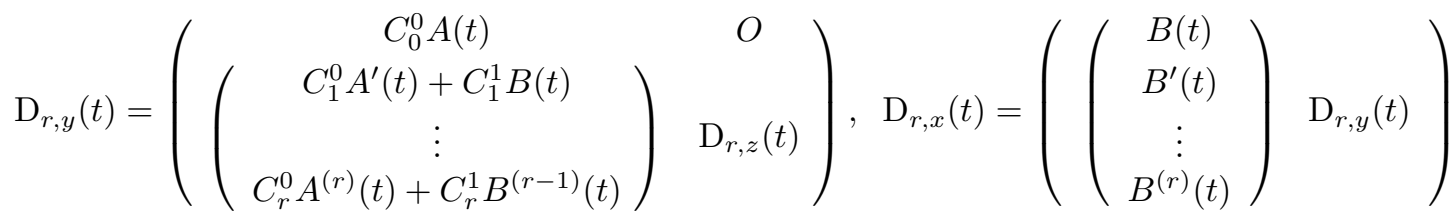

are analogs for the matrices $\Gamma_{r, z}, \Gamma_{r, y}$ and $\Gamma_{r, x}$ applied to DAE (3.9). They have dimensions $n(r+1) \times n r, \quad n(r+1) \times n(r+1)$ and $n(r+1) \times n(r+2)$, respectively; $C_{i}^{j}=\frac{i !}{j !(i-j) !}$ are binomial coefficients.

Suppose that the elements of the matrices $A(t)$ and $B(t)$ are enough times continuously differentiable on $I$ functions. In addition, let us assume that for some $r: 0 \leqslant r \leqslant n$ the condition $\operatorname{rank} \mathrm{D}_{r, z}(t)=\rho=$ const $\forall t \in I$ holds and there is non-special minor of the $n(r+1)$ order in the matrix $\mathrm{D}_{r, x}(t) \forall t \in I$. It includes $\rho$ columns of the matrix $\mathrm{D}_{r, z}(t)$ and the first $n$ columns of the matrix $\mathrm{D}_{r, y}(t)$. Let us introduce $d=n r-\rho$.

\footnotetext{
${ }^{\ddagger}$ Here and below we use the notation: $\|*\|$ is one of the norms in Euclidean space.
} 
Definition 7 ( [5], p. 76). Non-special minor of the $n(r+1)$ order $\forall t \in I$ of the matrix $\mathrm{D}_{r, x}(t)$, which includes $\rho$ columns of the matrix $\mathrm{D}_{r, z}(t)$ and the first $n$ columns of the matrix $\mathrm{D}_{r, y}(t)$ is called the resolving minor.

Let us assume that we know exactly which columns of the matrix $\mathrm{D}_{r, x}(t)$ are included into resolving minor. We delete $n-d$ columns of the matrix $\bar{B}(t)=\operatorname{colon}\left(B(t), B^{\prime}(t), \ldots, B^{(r)}(t)\right)$, which are not included in the resolving minor. After the appropriate column permutation of $\mathrm{D}_{r, x}(t)$ we obtain the matrix

$$
\Lambda_{r}(t)=\mathrm{D}_{r, x}(t) \operatorname{diag}\left(Q^{-1}\left(\begin{array}{c}
O \\
E_{d}
\end{array}\right), Q^{-1}, \ldots, Q^{-1}\right) .
$$

Let us note that $\operatorname{diag}\left(A_{1}, A_{2}, \ldots, A_{s}\right)$ means quasi-diagonal matrix with the blocks on the main diagonal, other elements are equal to zero, $E_{d}$ is the identity matrix of order $d, Q$ is $(n \times n)$ permutation matrix.

Definition 8 ( [6], p. 62). The system of linear algebraic equations

$$
\mathrm{D}_{r, x}(t) \operatorname{colon}\left(x, y, z_{1}, \ldots, z_{r}\right)=0
$$

is called $r$-derivative array equations of system (3.9), where $x, y, z_{j} \in \mathbf{R}^{n}$.

It was shown $[6$, p. 63] that under some assumptions there exists unique linear differential operator

$$
\mathcal{R}=R_{0}(t)+R_{1}(t) \frac{d}{d t}+\ldots+R_{r}(t)\left(\frac{d}{d t}\right)^{r}
$$

with continuous on $I$ coefficients that converts system (3.9) into the form

$$
\begin{aligned}
& x_{1}^{\prime}(t)+J_{1}(t) x_{1}(t)=0, \\
& x_{2}(t)+J_{2}(t) x_{1}(t)=0,
\end{aligned}
$$

where $\operatorname{colon}\left(x_{1}(t), x_{2}(t)\right)=Q x(t)$ and $x_{1} \in \mathbf{R}^{n-d}, x_{2} \in \mathbf{R}^{d}$.

Then function (3.10) takes the form

$$
y(t)=C_{1}(t) x_{1}(t)+C_{2}(t) x_{2}(t),
$$

where $\left(\begin{array}{c}C_{1}(t) \\ C_{2}(t)\end{array}\right)=C(t) Q^{-1}$. Thus, the output function

$$
y_{1}(t)=C_{1}(t) x_{1}(t)
$$

corresponds to system $(3.12)$.

Wherein $(n \times n)$-matrices $R_{j}(t)$ are uniquely determined by the resolving minor as

$$
\left(\begin{array}{llll}
R_{0}(t) & R_{1}(t) & \ldots & R_{r}(t)
\end{array}\right)=\left(\begin{array}{llll}
E_{n} & O & \ldots & O
\end{array}\right) \Lambda_{r}^{\top}(t)\left(\Lambda_{r}(t) \Lambda_{r}^{\top}(t)\right)^{-1} .
$$

The coefficients of system (3.12), (3.13) are determined from the relation

$$
\left(\begin{array}{cc}
J_{2}(t) & E_{d} \\
J_{1}(t) & O
\end{array}\right)=\left(\begin{array}{llll}
R_{0}(t) R_{1}(t) & \ldots & \left.R_{r}(t)\right) \bar{B}(t) Q^{-1}
\end{array}\right.
$$

Theorem 2 ( [6]). Let us assume that

1) $A(t), B(t) \in \mathbf{C}^{2 r+1}(I)$;

2) $\operatorname{rank} \mathrm{D}_{r, z}(t)=\rho=$ const $\forall t \in I$;

3) there is the resolving minor in the matrix $\mathrm{D}_{r, x}(t)$;

4) $\operatorname{rank} \mathrm{D}_{r+1, y}(t)=\operatorname{rank} \mathrm{D}_{r, y}(t)+n \forall t \in I$.

Then every solution of (3.9) is the solution of (3.12), (3.13) and vice versa. 
Definition 9 ( [6], p.64). System (3.12), (3.13) is called the equivalent form for DAE (3.9).

Corollary 1 ( [6]). Let us suppose that all assumptions of the Theorem 2 are satisfied. Then problem (3.8), (3.9) is solvable if and only if

$$
x_{2,0}+J_{2}\left(t_{0}\right) x_{1,0}=0
$$

where $\operatorname{colon}\left(x_{1,0}, x_{2,0}\right)=Q z_{0}$. Moreover, if a solution to problem (3.8), (3.9) exists then it is unique.

Definition 10 ( [5], p. 78). Initial condition (3.8) which satisfies (3.17) is called consistent with system (3.9).

Let us return to DAE (1.1) and suppose that all assumptions of the Theorem 1 are fulfilled. Property (1.3) ensures that $f_{1}(t, 0,0)=0, f_{0}(t, 0,0)=0 \quad \forall t \in I_{0}$ in system (3.1), (3.2).

Let us construct the system of the first approximation for DAEs (3.1), (3.2)

$$
\begin{gathered}
x_{1}^{\prime}(t)+\tilde{J}_{1}(t) x_{1}(t)=0, \\
x_{2}(t)+\tilde{J}_{2}(t) x_{1}(t)=0, \quad t \in I_{0},
\end{gathered}
$$

where $\tilde{J}_{1}(t)=-\frac{\partial f_{1}}{\partial x_{1}}(t, 0,0), \quad \tilde{J}_{2}(t)=-\frac{\partial f_{0}}{\partial x_{1}}(t, 0,0)$.

The following theorem states that operations of linearization and transition to equivalent form are commuted [6].

Theorem 3. Let us assume that

1) $F(t, x, y) \in \mathbf{C}^{2 r+2}(\mathcal{D})$;

2) assumptions 2), 3) of Theorem 1 are satisfied.

Then systems (3.12), (3.13) and (3.18), (3.19) coincide on some sufficiently small interval $I_{0}=\left(a_{0}-\varepsilon_{0}, a_{0}+\varepsilon_{0}\right) \subseteq I, \quad 0<\varepsilon_{0} \leqslant \varepsilon$.

\section{Local R-observability}

Let us consider system (3.1) which is a system of ODE in the normal form.

Definition 11 ( [3], p.366). System (3.1) is called locally observable in terms of output (3.5) on the interval $T=\left[t_{0}, t_{1}\right] \subset I_{0}$ if there exists a number $\delta_{1}>0$ that for any two distinct points $x_{1,0}, \tilde{x}_{1,0} \in \mathbf{R}^{n-d}$ from the $\delta_{1}$-neighborhood of the point $x_{1}=0$ the relation

$$
\phi_{1}\left(t, x_{1}\left(t, t_{0}, x_{1,0}\right)\right) \not \equiv \phi_{1}\left(t, x_{1}\left(t, t_{0}, \tilde{x}_{1,0}\right)\right), \quad t \in T,
$$

is fulfilled, where $x_{1}\left(t, t_{0}, x_{1,0}\right)$ is a solution of system (3.1) with the initial condition $x_{1}\left(t_{0}\right)=$ $x_{1,0}$.

Let us use the definition of the R-observability [2, p. 41] for a linear system of DAEs and reformulate it in terms of the present paper.

Definition 12. System (3.9), (3.10) is called R-observable on the interval $T$ if it is possible to uniquely restore the initial state of the system in terms of function $y(t)$ and this state is consistent.

With this in mind, we can define the local $R$-observability for a nonlinear system of DAEs. 
Definition 13. System (1.1), (1.2) is called locally R-observable on the interval $T$ if there exists a number $\delta>0$ such that for any two consistent initial states $z_{0}, \tilde{z}_{0} \in \mathbf{R}^{n}$ from $\delta$-neighborhood of the point $x=0$ the relation

$$
\phi\left(t, x\left(t, t_{0}, z_{0}\right)\right) \not \equiv \phi\left(t, x\left(t, t_{0}, \tilde{z}_{0}\right)\right), \quad t \in T,
$$

is fulfilled, where $x\left(t, t_{0}, z_{0}\right)$ is a solution of system (1.1) with the initial condition $x\left(t_{0}\right)=z_{0}$.

Let us formulate the condition of the R-observability for DAEs (3.9), (3.10) obtained in [5]. To do this, we define the observability matrix for system (3.12), (3.14)

$$
\operatorname{colon}\left(\mathcal{S}_{0}(t) \mathcal{S}_{1}(t) \quad \ldots \quad \mathcal{S}_{n-d-1}(t)\right),
$$

where $\mathcal{S}_{0}(t)=C_{1}(t), \quad \mathcal{S}_{i}(t)=-\mathcal{S}_{i-1}(t) J_{1}(t)+\mathcal{S}_{i-1}^{\prime}(t), \quad i=1, \ldots, n-d-1$.

Lemma 1. Let us suppose that all assumptions of the Theorem 2 are satisfied. System (3.9), (3.10) is $R$-observable on the interval $T$ if there exists such point $\sigma \in T$ that relation

$$
\operatorname{rank}\left(\operatorname{colon}\left(\mathcal{S}_{0}(\sigma) \mathcal{S}_{1}(\sigma) \quad \ldots \quad \mathcal{S}_{n-d-1}(\sigma)\right)\right)=n-d
$$

is fulfilled.

Consider the well-known result for ODEs [3, p. 366].

Theorem 4. If the system of a linear approximation (3.12) is completely observable via the output (3.14) on the interval $T$, then the nonlinear system (3.1) is locally observable via the output (3.5) in this interval.

The following theorem is a sufficient condition for the local $R$-observability of the nonlinear system of DAEs (1.1), (1.2).

Theorem 5. Let us assume that

1) $F(t, x, y) \in \mathbf{C}^{2 r+2}(\mathcal{D})$;

2) assumptions 2), 3) of Theorem 1 are satisfied.

If system of the linear approximation (3.9), (3.10) is R-observable on the interval $T$ then nonlinear system (1.1), (1.2) is locally R-observable on this interval.

Proof. Let us assume that system of the linear approximation (3.9), (3.10) is $R$-observable on the interval $T$. By Definition 12 this means that for any two different output functions $y(t)$ and $\tilde{y}(t)$ consistent initial states $z_{0}, \tilde{z}_{0} \in \mathbf{R}^{n}$ which correspond to the solutions $x\left(t, t_{0}, z_{0}\right)$ and $x\left(t, t_{0}, \tilde{z}_{0}\right)$ are not coincident on $T$. Taking into account (3.14), we have

$$
y_{1}(t)=C_{1}(t) x_{1}\left(t, t_{0}, x_{1,0}\right) \neq \tilde{y}_{1}(t)=C_{1}(t) x_{1}\left(t, t_{0}, \tilde{x}_{1,0}\right), \quad t \in T,
$$

where $x_{1}\left(t, t_{0}, x_{1,0}\right)=\left(E_{n-d} O\right) Q x\left(t, t_{0}, z_{0}\right), x_{1}\left(t, t_{0}, \tilde{x}_{1,0}\right)=\left(E_{n-d} O\right) Q x\left(t, t_{0}, \tilde{z}_{0}\right)$ are solutions of system (3.12) with initial conditions $x_{1}\left(t_{0}\right)=x_{1,0}$ and $x_{1}\left(t_{0}\right)=\tilde{x}_{1,0}$, respectively $\left(x_{1,0}, \tilde{x}_{1,0} \in \mathbf{R}^{n-d}\right)$. This means the full observability of system (3.12), (3.14) [3, p. 366]. Thus, the $R$-observability of system (3.9), (3.10) is equivalent to the full observability of DAEs (3.12), (3.14).

Let us consider system (1.1). Assumptions 1)-3) of Theorem 5 provide equivalence in the sense of solutions between systems (1.1) and (3.1), (3.2). According to the Theorem 4, the full observability of system (3.12), (3.14) implies the local observability of nonlinear system (3.1), (3.5) on the same interval $(T)$. This means that there exists a number $\delta_{1}>0$ that for any two different $x_{1,0}, \tilde{x}_{1,0} \in \mathbf{R}^{n-d}$ from $\delta_{1}$-neighborhood of the point $x_{1}=0$ the relation

$$
\phi_{1}\left(t, x_{1}\left(t, t_{0}, x_{1,0}\right)\right) \not \equiv \phi_{1}\left(t, x_{1}\left(t, t_{0}, \tilde{x}_{1,0}\right)\right), \quad t \in T
$$


is satisfied.

Then, taking into account expressions (3.4), (3.5), we obtain

$$
\phi\left(t, x\left(t, t_{0}, z_{0}\right)\right)=\operatorname{colon}\left(\phi_{1}\left(t, x_{1}\left(t, t_{0}, x_{1,0}\right)\right), \phi_{2}\left(t, x_{1}\left(t, t_{0}, x_{1,0}\right)\right),\right.
$$

where $x_{1,0}$ is determined from $z_{0}=Q^{-1} \operatorname{colon}\left(x_{1,0}, x_{2,0}\right)$,

$$
\phi\left(t, x\left(t, t_{0}, \tilde{z}_{0}\right)\right)=\operatorname{colon}\left(\phi_{1}\left(t, x_{1}\left(t, t_{0}, \tilde{x}_{1,0}\right)\right), \phi_{2}\left(t, x_{1}\left(t, t_{0}, \tilde{x}_{1,0}\right)\right),\right.
$$

where $\tilde{x}_{1,0}$ is determined from $\tilde{z}_{0}=Q^{-1} \operatorname{colon}\left(\tilde{x}_{1,0}, \tilde{x}_{2,0}\right)$,

Taking into account expressions (4.3) and (4.4), we find from (4.2) that relation

$$
\phi\left(t, x\left(t, t_{0}, z_{0}\right)\right) \not \equiv \phi\left(t, x\left(t, t_{0}, \tilde{z}_{0}\right)\right), \quad t \in T
$$

is independent of the values $\phi_{2}\left(t, x_{1}\left(t, t_{0}, x_{1,0}\right)\right)$ and $\phi_{2}\left(t, x_{1}\left(t, t_{0}, \tilde{x}_{1,0}\right)\right)$.

It is obvious that $x_{2,0}, \tilde{x}_{2,0}$ can be chosen so that $z_{0}$ and $\tilde{z}_{0}$ are consistent and they belong to $\delta_{1}$-neighborhood of the point $x=0$. Thus, it is shown that there exists a number $\delta=\delta_{1}>0$ that for any consistent initial conditions $z_{0}, \tilde{z}_{0}$ from $\delta$-neighborhood of the point $x=0$ relation (4.5) is satisfied. This means the local $R$-observability of system (1.1), (1.2).

Corollary 2. Let us suppose that all assumptions of Theorem 2 are satisfied. If system (3.9), (3.10) is R-observable on the interval $T$ then it is locally $R$-observable on this interval.

The validity of the corollary follows directly from the proof of Theorem 5 .

\section{Example}

Consider the nonlinear system that describe the Belousov-Zhabotinsky chemical reaction:

$$
\left(\begin{array}{lll}
1 & 0 & 0 \\
0 & 1 & 0 \\
0 & 0 & 0
\end{array}\right)\left(\begin{array}{c}
\chi_{1}^{\prime}(t) \\
\chi_{2}^{\prime}(t) \\
\chi_{3}^{\prime}(t)
\end{array}\right)-\left(\begin{array}{c}
-0.8 \chi_{1}(t)+10 \chi_{2}(t)-0.6 \chi_{1}(t) \chi_{3}(t) \\
-10 \chi_{2}(t)+1.6 \chi_{3}(t) \\
0.8 \chi_{1}(t)+1.6 \chi_{3}(t)-0.6 \chi_{1}(t) \chi_{3}(t)
\end{array}\right)=0,
$$

where $t \in \mathbf{R}_{+}=[0,+\infty), x(t)=\operatorname{colon}\left(\chi_{1}(t), \chi_{2}(t), \chi_{3}(t)\right) \quad\left(\chi_{i}(i=1,2,3): \mathbf{R}_{+} \rightarrow \mathbf{R}\right)$ is the unknown function of state. The initial condition is $x\left(a_{0}\right)=0, a_{0} \in(0,+\infty)$.

Let us define the output function for (5.1) as

$$
y(t)=\left(\begin{array}{ccc}
e^{t} & 0 & 2 e^{t} \\
t & 0 & 2 \cos t
\end{array}\right)\left(\begin{array}{l}
\chi_{1}(t) \\
\chi_{2}(t) \\
\chi_{3}(t)
\end{array}\right)+\left(\begin{array}{c}
0 \\
e^{\chi_{2}(t)}-1
\end{array}\right) .
$$

Let us show that system (5.1), (5.2) is locally $R$-observable in the neighborhood $A_{\varepsilon}$ of the point $a_{0}$. To do this, we verify that assumptions of Theorem 5 are satisfied.

Assumption 1) is obviously satisfied for all $r$. To check the assumptions 2), 3) we construct two derivative array equations of system (5.1)

$$
\begin{gathered}
\left(\begin{array}{c}
\chi_{1}^{\prime \prime}+0.8 \chi_{1}^{\prime}-10 \chi_{2}^{\prime}+0.6 \chi_{1}^{\prime} \chi_{3}+0.6 \chi_{1} \chi_{3}^{\prime} \\
\chi_{2}^{\prime \prime}+10 \chi_{2}^{\prime}-1.6 \chi_{3}^{\prime} \\
-0.8 \chi_{1}^{\prime}-1.6 \chi_{3}^{\prime}+0.6 \chi_{1}^{\prime} \chi_{3}+0.6 \chi_{1} \chi_{3}^{\prime}
\end{array}\right)=0, \\
\left(\begin{array}{c}
\left.\chi_{1}^{\prime \prime \prime}+0.8 \chi_{1}^{\prime \prime}-10 \chi_{2}^{\prime \prime}+0.6 \chi_{1}^{\prime \prime} \chi_{3}+0.6 \chi_{1} \chi_{3}^{\prime \prime}+1.2 \chi_{1}^{\prime} \chi_{3}^{\prime}\right) \\
\chi_{2}^{\prime \prime \prime}+10 \chi_{2}^{\prime \prime}-1.6 \chi_{3}^{\prime \prime} \\
-0.8 \chi_{1}^{\prime \prime}-1.6 \chi_{3}^{\prime \prime}+0.6 \chi_{1}^{\prime \prime} \chi_{3}+0.6 \chi_{1} \chi_{3}^{\prime \prime}+1.2 \chi_{1}^{\prime} \chi_{3}^{\prime}
\end{array}\right)=0
\end{gathered}
$$


and matrix

$$
\Gamma_{2, x}=\left(\begin{array}{ccc|c|ccc|cc|cc|ccc}
P_{1} & -10 & 0.6 \chi_{1} & 1 & 0 & 0 & 0 & 0 & 0 & 0 & 0 & 0 \\
0 & 10 & -1.6 & 0 & 1 & 0 & 0 & 0 & 0 & 0 & 0 & 0 \\
P_{2} & 0 \\
0.6 \chi_{3}^{\prime} & 0 & P_{3} & 0 & 0 & 0 & 0 & 0 \\
0 & 0 & 0.6 \chi_{1}^{\prime} & P_{1} & -10 & 0.6 \chi_{1} & 1 & 0 & 0 & 0 & 0 & 0 & 0 \\
\hline 0.6 \chi_{3}^{\prime} & 0 & 0.6 \chi_{1}^{\prime} & 0 & 10 & -1.6 & 0 & 1 & 0 & 0 & 0 \\
0 & & 0 & P_{3} & 0 & 0 & 0 & 0 & 0 & 0 \\
\hline \hline 0.6 \chi_{3}^{\prime \prime} & 0 & 0.6 \chi_{1}^{\prime \prime} & 1.2 \chi_{3}^{\prime} & 0 & 1.2 \chi_{1}^{\prime} & P_{1} & -10 & 0.6 \chi_{1} & 1 & 0 & 0 \\
0 & 0 & 0 & 0 & 0 & 0 & 0 & 10 & -1.6 & 0 & 1 & 0 \\
0.6 \chi_{3}^{\prime \prime} & 0 & 0.6 \chi_{1}^{\prime \prime} & 1.2 \chi_{3}^{\prime} & 0 & 1.2 \chi_{1}^{\prime} & P_{2} & 0 & P_{3} & 0 & 0 & 0
\end{array}\right),
$$

where $P_{1}=0.8+0.6 \chi_{3}, P_{2}=-0.8+0.6 \chi_{3}, P_{3}=-1.6+0.6 \chi_{1}$.

The matrix $\Gamma_{2, x}$ divided into four blocks by two double lines, the upper left block is $\Gamma_{1, x}$. It is obvious that $\operatorname{rank} \Gamma_{1, x}\left(\alpha_{1}\right)=n(r+1)=6\left(\alpha_{1}=\left(a_{0}, 0, \ldots, 0\right) \in \mathbf{R}^{1+3 n+2 l}\right)$. Columns that are included into the resolving minor are framed in the matrix $\Gamma_{1, x}: \rho=2$ columns of the matrix $\Gamma_{1, z}\left(\alpha_{1}\right), n=3$ first columns of the matrix $\Gamma_{1, y}\left(\alpha_{1}\right)$ and the third column of the matrix $\Gamma_{1, x}$. Thus, for 1-derivative array equations (5.1), (5.3) all assumptions of an implicit function theorem are fulfilled $[7, \mathrm{p} .66]$. Then $n(r+1)=6$ components of the vector colon $\left(x, y, z_{1}\right)$ can be expressed from (5.1), (5.3) (they are denoted by $\xi$ ) as a function of $t$ and other $n=3$ components of this vector (they are denoted by $\eta$, see $(2.2))$. Therefore, $\xi=\left(\chi_{3}, \chi_{1}^{\prime}, \chi_{2}^{\prime}, \chi_{3}^{\prime}, \chi_{1}^{\prime \prime}, \chi_{2}^{\prime \prime}\right), \eta=\left(\chi_{1}, \chi_{2}, \chi_{3}^{\prime \prime}\right)$. These functions are too cumbersome and they are not presented here.

It is obvious that $\mathcal{F}_{2}\left(\alpha_{2}\right)=0$, where $\alpha_{2}=\left(a_{0}, 0, \ldots, 0\right) \in \mathbf{R}^{1+4 n}$. In this case the matrix $\bar{\Gamma}_{1, z}$ which is obtained by substituting functions $\xi$ into $\Gamma_{1, z}$ is constant and it coincides with the matrix $\Gamma_{1, z}$. Thus, rank $\bar{\Gamma}_{1, z}=\rho=2$ everywhere in $\mathcal{W}$.

The matrix $\Gamma_{2, y}$ consists of the last 9 columns of the matrix $\Gamma_{2, x}$. It is easy to verify that $\operatorname{rank} \Gamma_{2, y}\left(\alpha_{2}\right)=\operatorname{rank} \Gamma_{1, y}\left(\alpha_{1}\right)+n=5+3=8$.

Thus, assumptions 2 ) and 3 ) of Theorem 5 are satisfied.

Let us construct the system of the linear approximation for DAE (5.1) and function (5.2)

$$
\begin{gathered}
\left(\begin{array}{lll}
1 & 0 & 0 \\
0 & 1 & 0 \\
0 & 0 & 0
\end{array}\right)\left(\begin{array}{l}
\chi_{1}^{\prime}(t) \\
\chi_{2}^{\prime}(t) \\
\chi_{3}^{\prime}(t)
\end{array}\right)+\left(\begin{array}{ccc}
0.8 & -10 & 0 \\
0 & 10 & -1.6 \\
-0.8 & 0 & -1.6
\end{array}\right)\left(\begin{array}{l}
\chi_{1}(t) \\
\chi_{2}(t) \\
\chi_{3}(t)
\end{array}\right)=0 \\
y(t)=\left(\begin{array}{ccc}
e^{t} & 0 & 2 e^{t} \\
t & 1 & 2 \cos t
\end{array}\right)\left(\begin{array}{l}
\chi_{1}(t) \\
\chi_{2}(t) \\
\chi_{3}(t)
\end{array}\right) .
\end{gathered}
$$

In order to show that system $(5.4),(5.5)$ is $R$-observable we use Lemma 1 . To do this, we verify that assumptions of Theorem 2 are fulfilled for $r=1$.

It is obvious that Assumption 1) is true. To verify assumptions 2)-4) for DAE (5.4) we construct the matrix

$$
\mathrm{D}_{2, x}(t)=\left(\begin{array}{cc|c|c|ccc|cc||cc|ccc}
0.8 & -10 & 0 & 1 & 0 & 0 & 0 & 0 & 0 & 0 & 0 & 0 \\
0 & 10 & -1.6 & 0 & 1 & 0 & 0 & 0 & 0 & 0 & 0 & 0 \\
-0.8 & 0 & -1.6 & 0 & 0 & 0 & 0 & 0 \\
0 & 0 & 0 & 0.8 & -10 & 0 & 1 & 0 & 0 & 0 & 0 \\
\hline 0 & 0 & 0 & 0 & 10 & -1.6 & 0 & 1 & 0 & 0 & 0 & 0 \\
0 & 0 & 0 & -0.8 & 0 & -1.6 & 0 & 0 & 0 & 0 & 0 & 0 \\
\hline 0 & 0 & 0 & 0 & 0 & 0 & 0.8 & -10 & 0 & 1 & 0 & 0 \\
0 & 0 & 0 & 0 & 0 & 0 & 0 & 10 & -1.6 & 0 & 1 & 0 \\
0 & 0 & 0 & 0 & 0 & 0 & -0.8 & 0 & -1.6 & 0 & 0 & 0
\end{array}\right) .
$$


It is easy to calculate that $\operatorname{rank} \mathrm{D}_{1, z}(t)=\rho=2 \forall t \in \mathbf{R}_{+}$.

The matrix $\mathrm{D}_{2, x}(t)$ is divided into four blocks by double line and the upper left block is $\mathrm{D}_{1, x}(t)$. Columns which are included into the resolving minor are framed in the matrix $\mathrm{D}_{1, x}(t)$. Thus, rank $\mathrm{D}_{1, x}(t)=n(r+1)=6$ and resolving minor includes $\rho=2$ columns of the matrix $\mathrm{D}_{1, z}(t), n=3$ first columns of the matrix $\mathrm{D}_{1, y}(t)$ and the third column of the matrix $\mathrm{D}_{1, x}(t)$. The matrix $\mathrm{D}_{2, y}(t)$ consists of the last 9 columns of the matrix $\mathrm{D}_{2, x}(t)$. So the assumption 4$)$ of Theorem $2 \operatorname{rank} \mathrm{D}_{2, y}(t)=\operatorname{rank} \mathrm{D}_{1, y}(t)+n=5+3=8$ is also satisfied.

Thus, all assumptions of Theorem 2 are fulfilled. To find the operator $\mathcal{R}$ of form (3.11) we write the matrix

$$
\Lambda_{1}(t)=\left(\begin{array}{ccccccc}
0 & 1 & 0 & 0 & 0 & 0 & 0 \\
-1.6 & 0 & 1 & 0 & 0 & 0 & 0 \\
-1.6 & 0 & 0 & 0 & 0 & 0 & 0 \\
0 & 0.8 & -10 & 0 & 1 & 0 & 0 \\
0 & 0 & 10 & -1.6 & 0 & 1 & 0 \\
0 & -0.8 & 0 & -1.6 & 0 & 0 & 0
\end{array}\right)
$$

and the column permutation matrix $Q$ in our case is the unit matrix.

According to (3.15), we find the coefficients of the operator $\mathcal{R}$

$$
\mathcal{R}=\left(\begin{array}{ccc}
0 & 0 & -0.625 \\
1 & 0 & 0 \\
0 & 1 & -1
\end{array}\right)+\left(\begin{array}{lll}
0 & 0 & 0 \\
0 & 0 & 0 \\
0 & 0 & 0
\end{array}\right) \frac{d}{d t}
$$

According to (3.16), we obtain

$$
\left(\begin{array}{cc}
J_{2}(t) & E_{1} \\
J_{1}(t) & O
\end{array}\right)=\left(\begin{array}{cc|c}
0.5 & 0 & 1 \\
\hline 0.8 & -10 & 0 \\
0.8 & 10 & 0
\end{array}\right) .
$$

Therefore, the equivalent system for DAE (5.4) has the form

$$
\begin{gathered}
x_{1}^{\prime}(t)+\left(\begin{array}{cc}
0.8 & -10 \\
0.8 & 10
\end{array}\right) x_{1}(t)=0, \\
x_{2}(t)+\left(\begin{array}{ll}
0.5 & 0
\end{array}\right) x_{1}(t)=0,
\end{gathered}
$$

where $x_{1}(t)=\left(\begin{array}{l}\chi_{1}(t) \\ \chi_{2}(t)\end{array}\right), x_{2}(t)=\chi_{3}(t)$. Thus

$$
y_{1}(t)=\left(\begin{array}{cc}
e^{t} & 0 \\
t & 1
\end{array}\right)\left(\begin{array}{l}
\chi_{1}(t) \\
\chi_{2}(t)
\end{array}\right) .
$$

To verify (4.1) for system (5.6), (5.8) we construct the observability matrix

$$
\left(\begin{array}{c}
\mathcal{S}_{0}(t) \\
\mathcal{S}_{1}(t)
\end{array}\right)=\left(\begin{array}{cc}
e^{t} & 0 \\
t & 1 \\
0.2 e^{t} & 10 e^{t} \\
0.2-0.8 e^{t} & 10 t-10
\end{array}\right)
$$

It is obvious that $\operatorname{rank}\left(\begin{array}{c}\mathcal{S}_{0}\left(a_{0}\right) \\ \mathcal{S}_{1}\left(a_{0}\right)\end{array}\right)=n-d=2 \quad \forall a_{0} \in(0,+\infty)$. Thus, all assumptions of Lemma 1 are fulfilled and system (5.4), (5.5) is $R$-observable in any neighborhood of the point $a_{0}$.

Thus, all assumptions of Theorem 5 are satisfied and it can be concluded that the nonlinear system of DAEs (5.1), (5.2) is locally $R$-observable in the neighborhood $A_{\varepsilon}$ of the point $a_{0}$.

This work was partially supported by the Russian Foundation for Basic Research (project no. 16-31-00101 мол_a) and by the Complex Program of Fundamental Scientific Research of the Siberian Branch of RAS (no. II.2) 


\title{
References
}

[1] K.E.Brenan, S.L.Campbell, L.R.Petzold, Numerical solution of initial-value problems in differential-algebraic equations, SIAM, 1996.

[2] L.Dai, Singular control system, Lecture notes in control and information sciences, vol. 118, Springer-Verlag, Berlin, Heidelberg, N.Y., 1989.

[3] I.V.Gaishun, Introduction to the theory of linear nonstationary systems, Natsional'naya Akademiya Nauk Belarusi, Institut Matematiki, 1999 (in Russian).

[4] M.Gerdin, Local identifiability and observability of nonlinear differantial-algebraic equations, Report no.: LiTH-ISY-R-2711, 14-th IFAC Symposium on System Identification, SYSID, 2006.

[5] A.A.Shcheglova, P.S.Petrenko, The R-observability and R-controllability of linear differential-algebraic systems, Izvestiya VUZ. Matematika, 56(2012), no. 3, 66-82.

[6] A.A.Shcheglova, Controllability of nonlinear algebraic differential systems, Automation and Remote Control, 69(2008), no. 10, 1700-1722.

[7] G.E.Shilov, Analysis. Part 1-2, Moscow, Nauka, 1972 (in Russian).

[8] F.R.Gantmacher, The theory of matrices, Chelsy Publishing Company, New York, 1959. .

[9] S.L.Campbell, L.R.Petzold, Canonical forms and solvable singular systems of differential equations, SIAM J. Alg. Disc. Meth., 4(1983), 517-522.

\section{Локальная R-наблюдаемость систем дифференциально- алгебраических уравнений}

Павел С. Петренко

Институт динамики систем и теории управления имени В.М. Матросова СО РАН

Лермонтова, 134, Иркутск, 664033

Russia

\begin{abstract}
Рассматривается система нелинейных обыкновенных дифференииалъных уравнений, не разрешенная относительно производной искомой вектор-функции и тождественно вырожденная в области определения. Допускается произвольно высокий индекс неразрешимости системы. Анализ проводится в предположениях, обеспечивающих существование структурной формы с разделенными "дифференциальной" и "алгебраической" подсистемами, которая эквивалентна исходной системе в смысле решений. Получены условия локалъной $R$-наблюдаемости такой системы по ее первому линейному приближению.
\end{abstract}

Ключевые слова: локальная наблюдаемость, дифференциально-алгебрачческие уравнения, наблюдаемая нелинейная система. 